\title{
Enhanced contrast detection of subsurface defects by pulsed infrared thermography based on the fourth order statistic moment, kurtosis
}

\author{
Francisco J. Madruga ${ }^{\mathrm{a}}$, Clemente Ibarra-Castanedo ${ }^{\mathrm{b}}$, Olga M Conde ${ }^{\mathrm{a}}$, Xavier P. Maldague ${ }^{\mathrm{b}}$, José M \\ López-Higuera ${ }^{\mathrm{a}}$. \\ ${ }^{a}$ Photonic Engineering Group, University of Cantabria, ETSIIT - Avda. Los Castros s/n, 39005 \\ Santander, Spain. \\ ${ }^{\mathrm{b}}$ Computer Vision And Systems Laboratory, Université Laval, G1V 0V6 Quebec City, Canada.
}

\begin{abstract}
The automatic detection of subsurface defects has become a desired goal in the application of non-destructive testing and evaluation techniques. In this paper, an algorithm based on the fourth order standardised statistic moment, i.e. kurtosis, is proposed for detection and/or characterization of subsurface defects having a thermal diffusivity either higher or lower than the host material. The analysis of thermographic data for the detection of defects can be reduced to the temporal statistics of the thermographic sequence. The final result provided by this algorithm is an image showing the different defects without the necessity of establishing other evaluating parameters such as the delayed time of the first image or the acquisition frequency in the analysis, which are required in other processing techniques. All the information is contained in a single image allowing to discriminate between the defect types (high o low thermal diffusivity). Synthetic data from Thermocalc ${ }^{\circledR}$ and experimental works using a Plexiglas ${ }^{\mathrm{TM}}$ specimen were performed showing good agreement. Processed results using synthetic and experimental data with other methods used in the field of thermography for defect detection and/or characterization are provided as well for comparison.
\end{abstract}

Keywords: pulsed thermography, image processing, higher-order statistics, kurtosis, defect detection.

\section{INTRODUCTION}

Thermal inspection is a useful technique for the non-destructive testing and evaluation (NDT\&E) of materials and systems. Active infrared thermography offers a reliable, straightforward and fast means for retrieving structural information from a specimen ${ }^{1}$. Concretely, the pulsed thermography technique is capable of detecting defects in large areas in a fast manner (a few seconds or minutes). The technique is based on the detection of surface temperature anomalies that appear in response to the application of a thermal pulse to the specimen surface. The temporal evolution of the surface temperature is recorded in a sequence of thermographic images (thermograms) using an infrared camera as it is shown in Figure 1.

Several processing techniques have been proposed in order to improve defect detection and quantification. For instance, thermal contrast-based techniques are very popular since they allow enhancing the defect contrast with simple subtraction operations ${ }^{1}$. The main drawbacks or this kind of algorithms are that they required to establish a non-defective area to calculate the thermal contrast, which is seldom easy or possible to do, and that they are strongly affected by nonuniform heating ${ }^{2}$. Some efforts have been directed to reduce these problems. The differential thermal contrast (DAC) is one example. Although automation is possible in some cases, the process requires the definition of thresholds that are established empirically. Moreover, there is no reduction on the amount of data to be processed.

A thermographic sequence is composed of several hundreds of thermograms. To provide an idea of the amount of data generated after a pulsed experiment, a thermogram matrix containing 1200 images on a 320x240 pixels configuration with a dynamic range of 14 bits, will require 175 Mbytes of storage space. There exist some signal processing algorithms that can help to compress the thermographic information contained on the sequence, many of them are based on the assumption that temperature profiles for non-defective pixels should follow the decay curve given by the onedimensional solution of the Fourier equation for a Dirac pulse ${ }^{3}$, i.e. Eq. (2). For example, the thermographic signal reconstruction (TSR) ${ }^{4}$ approach proposes to store only $n+1$ coefficients of an $n$ degree polynomial (typically four or five), which fit the decay curve of each pixel on a double-logarithmic space. Another recently proposed possibility ${ }^{5}$ is to

Thermosense XXXI, edited by Douglas D. Burleigh, Ralph B. Dinwiddie, Proc. of SPIE Vol. 7299

$72990 \mathrm{U} \cdot$ (C) $2009 \mathrm{SPIE} \cdot \mathrm{CCC}$ code: 0277-786X/09/\$18 · doi: 10.1117/12.818684

Proc. of SPIE Vol. 7299 72990U-1 
fit the thermographic data to an exponential curve, which has the advantage of compressing data even further since only two coefficients are obtained. Techniques based on matrix factorization ${ }^{6}$ or statistic parameter as in principal component thermography $(\mathrm{PCT})^{7}$ or using the skewness parameter ${ }^{8}$ have been reported and tested for solving NDT\&E limitations with success.

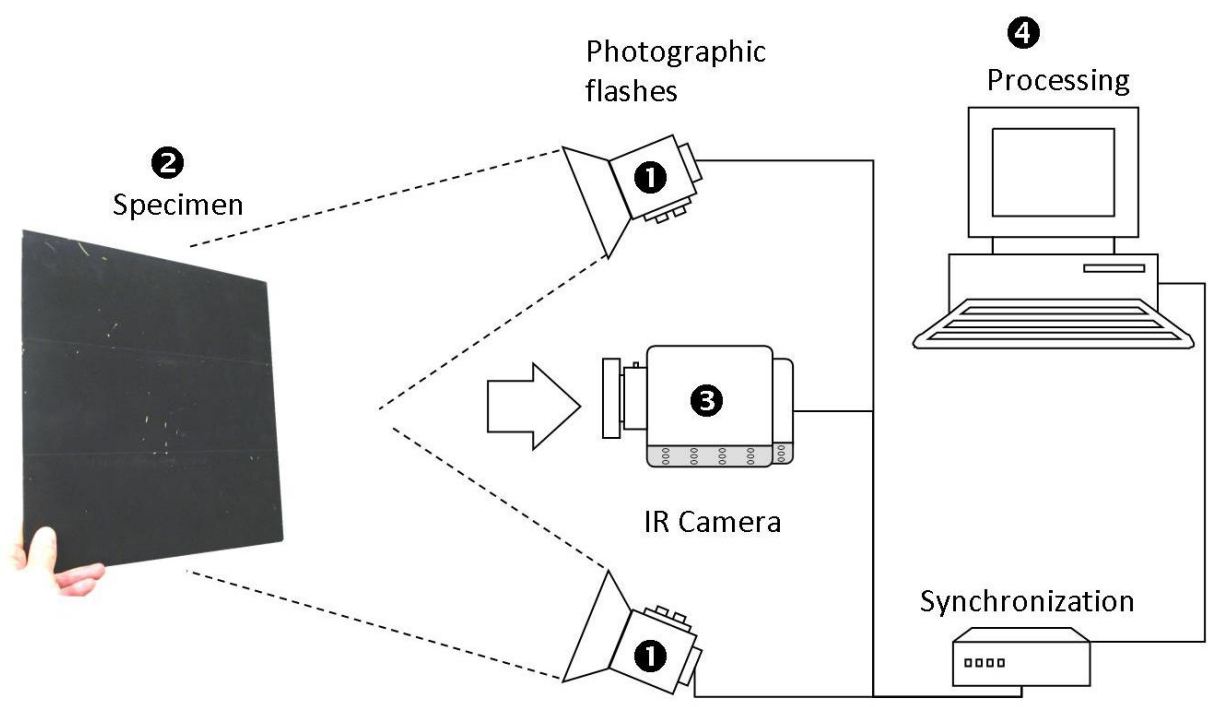

(a)

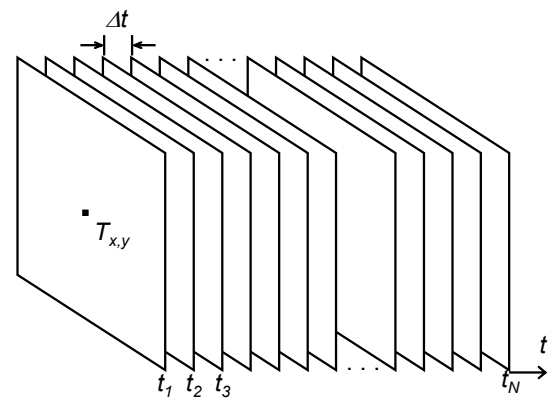

(b)

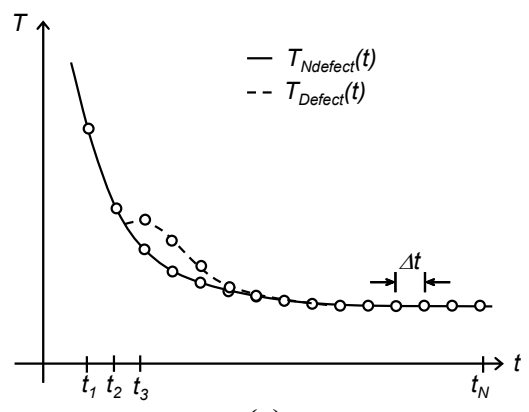

(c)

Figure 1. (a) Typical pulsed thermography setup, (b) thermogram sequence obtained from a pulsed inspection, and (c) temporal evolution of the surface temperature for a defective pixel (--) and a non-defective ( ) pixel.

The aim of this paper is to investigate the performance of the fourth order statistic parameter, namely kurtosis, applied to pulsed thermographic inspection. The kurtosis parameter have been used with synthetically generated data processing and they show enhanced defect contrast relative to unprocessed data and high compressing capabilities since the information contained in the unprocessed data sequence is compressed to a unique final image.

The following section discusses the use of the kurtosis parameter to analyze pulsed thermographic data.

\section{STATISTIC PARAMETER IN THERMOGRAPHIC INSPECTIONS}

The one dimensional solution of the Fourier equation for a Dirac delta function in a semi-infinite isotropic solid is given by: 


$$
T(z, t)=T_{0}+\frac{Q}{\sqrt{k \cdot \rho \cdot C_{p} \cdot \pi \cdot t}} \exp \left(-\frac{z^{2}}{4 \cdot \alpha \cdot t}\right)
$$

where $Q$ is the energy absorbed by the surface and $T_{0}$ is the initial temperature. For a thermographic inspection we must study the surface temperature evolution that is at the surface $\mathrm{z}=0$ and Eq. (1) can be rewritten as:

$$
T(z, 0)=T_{0}+\frac{Q}{\sqrt{k \cdot \rho \cdot C_{p} \cdot \pi \cdot t}}=T_{0}+\frac{Q}{e \sqrt{\pi \cdot t}}
$$

where $e \equiv\left(k \rho c_{\mathrm{P}}\right)^{1 / 2}[\mathrm{~m}]$ is the effusivity, which is a thermal property that measures the material ability to exchange heat with its surroundings.

Figure 1c shows the temperature decay curve described by Eq. (2) for a non-defective and a defective pixel. The decay curve for a non-defective pixel can be approximated as the square root of time whilst the profile for a defective pixel is different. The temperature evolution for both a defective and a non-defective pixel (sound pixel) can be represented as a statistical distribution of temperature values and different scores. For instance, using the data presented in Table 1, the histograms for defective and non-defective pixels can be reconstructed as shown in Figure 2. The difference of distribution can be described quantitatively using measures of variability or statistics.

Table 1. Table of score-frequency.

\begin{tabular}{l|ccccccccc}
\hline Scores & 0.15 & 0.16 & 0.17 & 0.18 & 0.19 & 0.20 & 0.21 & 0.22 & 0.23 \\
\hline Def. Frequency & 9 & 567 & 353 & 94 & 42 & 25 & 18 & 15 & 12 \\
Non-Def. Frequency & 580 & 379 & 64 & 34 & 25 & 18 & 15 & 12 & 11 \\
\hline
\end{tabular}
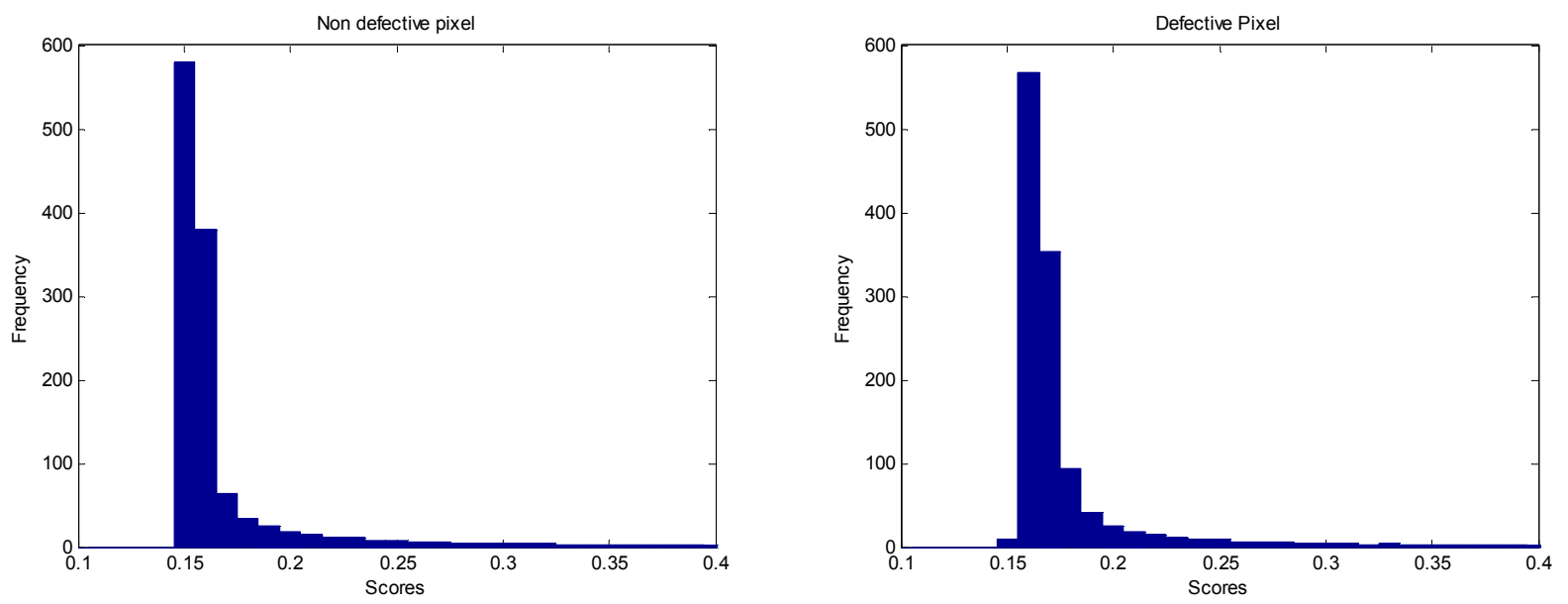

Figure 2. Histograms for (a) non-defective and (b) defective pixels.

The fourth standardized central moment, or kurtosis, is generally defined as a measure reflecting the degree to which a distribution is peaked. In particular, kurtosis provides information regarding the height of the distribution relative to the value of its standard deviation. Mathematically it is given by': 


$$
K=\frac{E\left[(X-E[X])^{4}\right]}{\sigma^{4}}
$$

where $E$ is the mathematical expectation and $\sigma$ is the standard deviation.

The kurtosis value can be classified in three general categories according to its derivation from a normal distribution: mesokurtic (normal distribution), leptokurtic (high degree of peakedness) and platykurtic (low degree of peakedness). Figure 3 illustrates these three distribution types. Their mean, variance and kurtosis values have been calculated with the data shown in Table 2. The distribution A, represented in Figure 3a, shows a quasi-normal distribution and its kurtosis value is near 3. The distribution B (Figure 3b) has the highest kurtosis value (more than 3 ) typical of a leptokurtic distribution, whilst the distribution C (Figure 3c) presents the smallest kurtosis value (less than 3 ) typical of a platykurtic distribution.
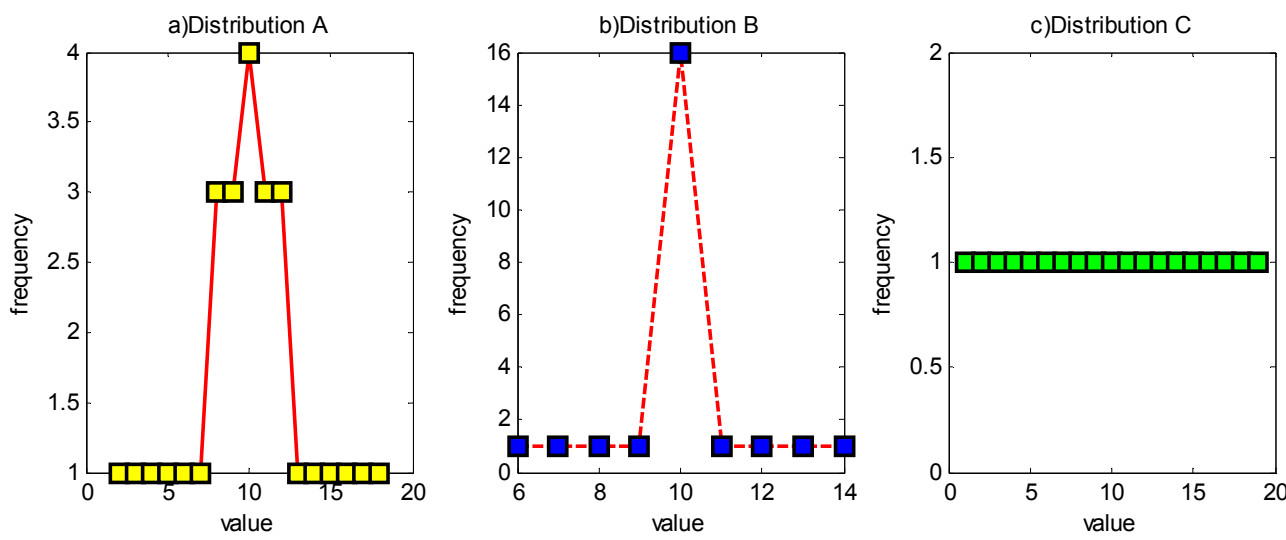

Figure 3. Example of (a) mesokurtic, (b) leptokurtic, and (c) platykurtic distributions.

Table 2. Example of normal (Distribution A) and peakedness and plainness (distributions B and C) distributions.

\begin{tabular}{ccccc}
\hline Distribution & Distribution Value & Mean & Variance & Kurtosis \\
\hline $\begin{array}{c}\text { A } \\
\text { mesokurtic }\end{array}$ & 23456788899910101010 & 10 & 15.8519 & 2.6920 \\
& 1111111212121314151617 & & & \\
\hline B & 6789101010101010101010 & 10 & 2.6087 & 4.7200 \\
leptokurtic & 1010101010101011121314 & & & \\
\hline $\begin{array}{c}\text { C } \\
\text { platykurtic }\end{array}$ & 123456789101112131415 & 10 & 31.6667 & 1.7933 \\
\hline
\end{tabular}

The surface temperature evolution for a non-defective area after pulsed thermography testing follows a leptokurtic distribution (Figure 3b), where ambient temperature presents the highest frequency. The kurtosis value in this case is very high. For a defective zone the surface temperature will present a higher or lower ambient temperature scores depending on the defective material diffusivity. For a defect having a higher thermal diffusivity than host material, the distribution is more peaked and the kurtosis value is higher for a defective pixel than for a non-defective pixel. On the contrary, the distribution has a wider peak and the kurtosis value is lower for a defective pixel than for a sound pixel for a defective material having a lower thermal diffusivity than the host material. Using this idea, it is possible to estimate the kurtosis values for every pixel in the thermogram matrix and to obtain an image with these values, a kurtogram. The kurtogram will provide an indication of, first, the location of eventual subsurface defects, and second, their thermal diffusivity. 


\section{SIMULATIONS}

In order to evaluate the dependence of the kurtosis value on the depth and thermal diffusivity of the defects, different thermography sequences where simulated using the Thermocal ${ }^{\circledR}$ software ${ }^{10}$. Three specimens containing six defects positioned as shown in Figure 4 have been simulated. The first two samples contained six defects at the same depth but having different defect material diffusivities: higher than the host material for the first specimen and lower than the host material for the second one. The third specimen has six defects at different depths and same defect material diffusivities (slightly higher than the host material). The characteristic of these samples are summarized in Table 3. One flash source centered on the surface is used to heat the sample for the simulation. Figure $4 \mathrm{~b}$ shows the raw thermograms obtained at different times. As can be observed from these images, it is possible to identify the non-uniform heating from the source.

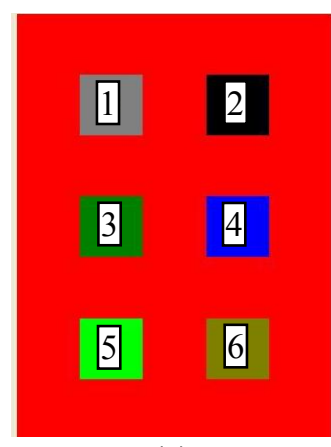

(a)

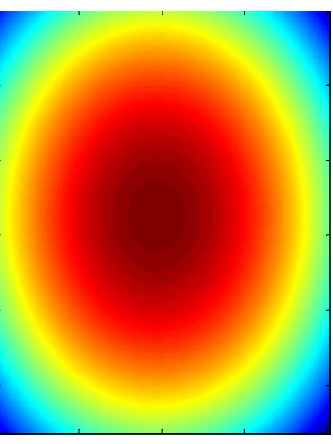

(b)

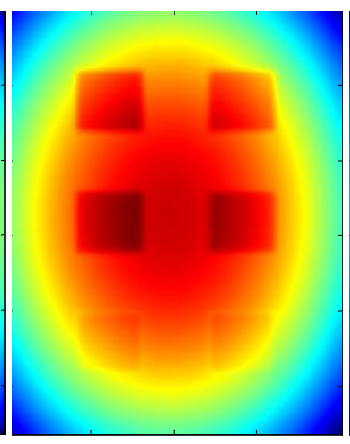

(c)

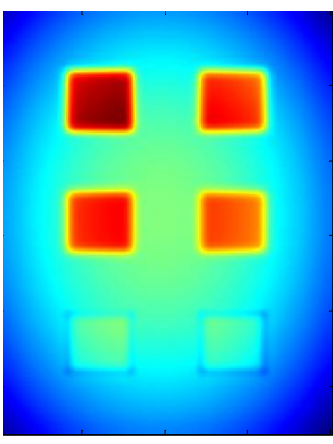

(d)

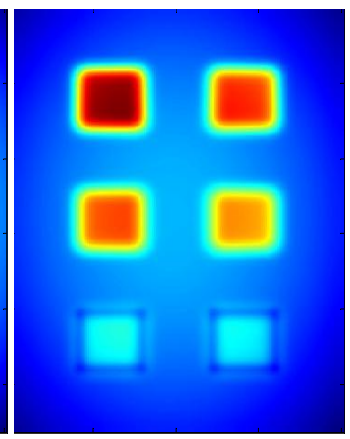

(e)

Figure 4. (a) Defect distribution in the specimens simulated with the Thermocal ${ }^{\circledR}$ software, and thermograms at $t=$ (b) $\mathrm{x} \mathrm{s}$ (first thermogram after heat stimulation), (c) x s, (d) x s, and (e) x s (last thermogram of the sequence).

Table 3. Characteristic of the simulated specimens in Figure 4.

\begin{tabular}{|c|c|c|c|c|c|c|c|c|c|c|c|c|}
\hline \multirow{3}{*}{ Specimen } & \multicolumn{12}{|c|}{ Defects } \\
\hline & \multicolumn{2}{|c|}{1} & \multicolumn{2}{|c|}{2} & \multicolumn{2}{|c|}{3} & \multicolumn{2}{|c|}{4} & \multicolumn{2}{|c|}{5} & \multicolumn{2}{|c|}{6} \\
\hline & $\begin{array}{c}\alpha \times 1^{-7} \\
{\left[\mathrm{~m}^{2} / \mathbf{s}\right]}\end{array}$ & $\underset{[\mathrm{mm}]}{z}$ & $\begin{array}{c}\alpha \times 1^{-7} \\
{\left[\mathbf{m}^{2} / \mathbf{s}\right]}\end{array}$ & $\underset{[\mathrm{mm}]}{z}$ & $\begin{array}{c}\alpha \times 1^{-7} \\
{\left[\mathrm{~m}^{2} / \mathrm{s}\right]}\end{array}$ & $\begin{array}{c}z \\
{[\mathbf{m m}]}\end{array}$ & $\begin{array}{c}\alpha \times 1^{-7} \\
{\left[\mathbf{m}^{2} / \mathbf{s}\right]}\end{array}$ & $\begin{array}{c}z \\
{[\mathbf{m m}]}\end{array}$ & $\begin{array}{c}\alpha \times 1^{-7} \\
{\left[\mathrm{~m}^{2} / \mathbf{s}\right]}\end{array}$ & $\underset{[\mathrm{mm}]}{z}$ & $\begin{array}{c}\alpha \times 1^{-7} \\
{\left[\mathrm{~m}^{2} / \mathrm{s}\right]}\end{array}$ & $\begin{array}{c}z \\
{[\mathrm{~mm}]}\end{array}$ \\
\hline 1 & 0.4 & 0.45 & 0.46 & 0.45 & 0.52 & 0.45 & 0.58 & 0.45 & 3 & 0.45 & 4 & 0.45 \\
\hline 2 & 0.4 & 0.45 & 0.34 & 0.45 & 0.28 & 0.45 & 0.22 & 0.45 & 0.05 & 0.45 & 0.04 & 0.45 \\
\hline 3 & 0.46 & 0.2 & 0.46 & 0.25 & 0.46 & 0.375 & 0.46 & 0.5 & 0.46 & 0.625 & 0.46 & 0.75 \\
\hline
\end{tabular}

*Host material: $\alpha=0.4 \times 10^{-7} \mathrm{~m}^{2} / \mathrm{s}$

Figure 5 shows the kurtosis value images for each specimen. As can be seen, in the case of simulated defects having equal to higher thermal diffusivity than the host material and a depth $z=0.45 \mathrm{~mm}$ (Figure $5 \mathrm{a}$ ), the kurtosis value is higher for defects (brighter) than for the host material (darker). On the contrary, for defects having equal to lower thermal diffusivity than the host material (Figure 5b), the kurtosis value is lower for defects (darker) than for the host material (brighter). Specimen 3 has simulated defects with a thermal diffusivity higher than the host material but they are located at different depths to analyze the impact of the depth on the kurtosis value. As can be seen in Figure 5c, defects deeper than $z=0.4 \mathrm{~mm}$ (defects 4 to 6) appear brighter than shallow ones (defects 1 to 3 ). From these results, it is suggested that the kurtosis value is a function of both, the depth and the thermal diffusivity of the material defect. 


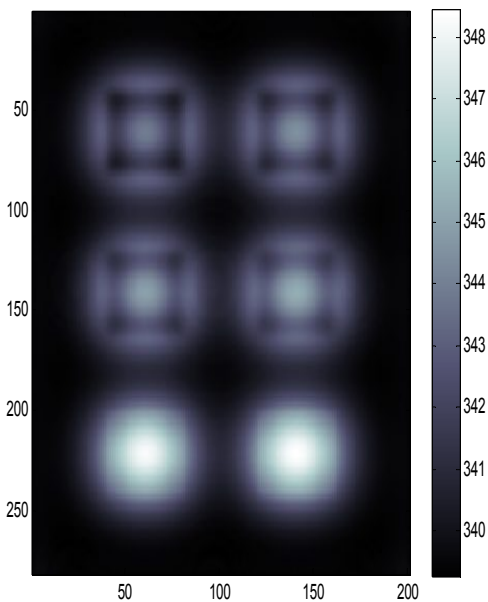

(a)

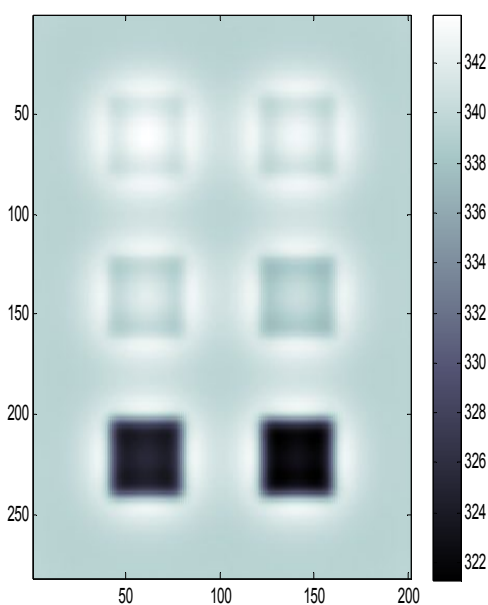

(b)

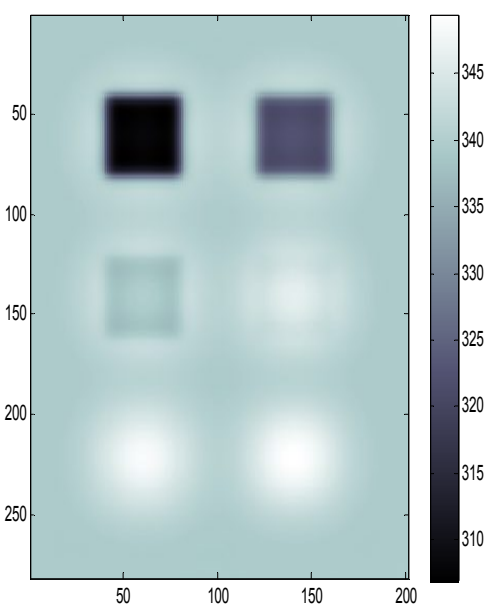

(c)

Figure 5. Kurtograms obtained from: (a) Specimen 1 with simulated defects of higher diffusivity than the host material, (b) Specimen 2 with simulated defects of lower diffusivity than the host material, and (c) Specimen 3 with simulated defects having a higher thermal diffusivity and several depths.

As a reference, Figure 6 shows the phasegrams at particular frequencies $(4.6 \mathrm{~Hz})$ obtained from pulsed phase thermography (PPT $)^{11}$. It is possible to estimate the depth of the defects using PPT data analyzing the phase profiles for every defect. The procedure is based on the determination of the blind frequency, i.e. the frequency at which a defect is detected. The blind frequency is a function of the depth of the defect. Hence, several phasegrams are needed to completely characterize a specimen with defects at different depths. On the contrary, the kurtograms contain all the defect information on a single image, from which, the relative quantification of the diffusivity and depth could be obtained.

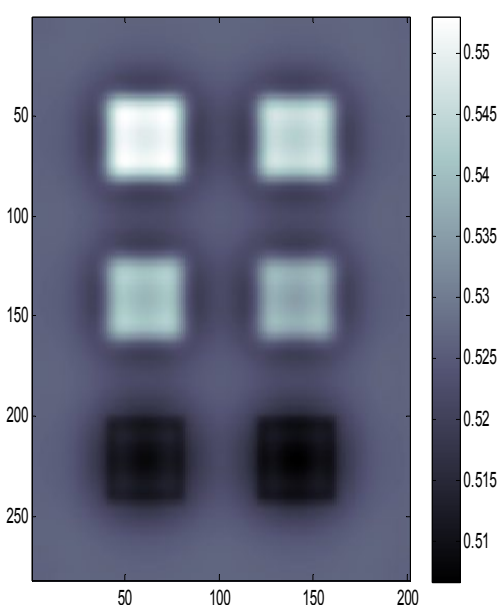

(a)

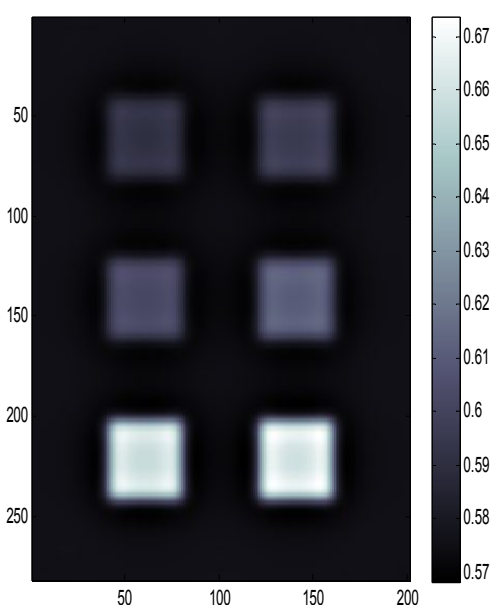

(b)

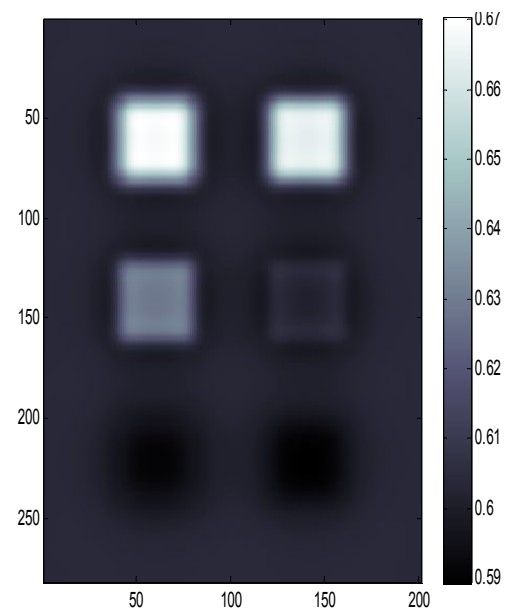

(c)

Figure 6. Phasegrams at $\mathrm{f}=4.6 \mathrm{~Hz}$ for: (a) Specimen 1, (b) Specimen 2, and (c) Specimen 3.

In the following section, an experimental Plexiglas ${ }^{\mathrm{TM}}$ plate was used to test the kurtosis technique on real data. More research is needed to derive experimental relationships and to validate them. 


\section{EXPERIMENTAL RESULTS}

A Plexiglas ${ }^{\text {TM }}$ sample $(25 \mathrm{~cm} \times 25 \mathrm{~cm} \times 6 \mathrm{~mm})$ with a flat-bottom slab $(16 \mathrm{~cm} \times 2 \mathrm{~cm})$ of constant depth $(3 \mathrm{~mm})$ was used to test the kurtosis method. A portion of the slab was covered with tin/lead based electronic solder to simulate a defect having a different (lower) thermal diffusivity than air.

The experimental configuration is depicted in Figure 1a. An FPA infrared camera (Santa Barbara Focalplane SBF125, 3 to $5 \mu \mathrm{m}$, with a $320 \times 256$ pixel array), working at a sampling frequency of $6 \mathrm{~Hz}$ was used. Two high power photographic flashes (Balcar FX 60), giving $6.4 \mathrm{~kJ}$ for $15 \mathrm{~ms}$ each, were used as heating sources. Reflection mode was used in all cases. Thermographic data was analyzed with a PC (Pentium 4, 2 GB RAM), and thermal data was processed using MATLAB $^{\circledR}$.

Figure 7 shows the results obtained using the kurtosis techniques (Figure 7c) and form others techniques for comparison: PPT phasegram (Figure 7c) and principal component thermography (PCT) (Figure 7b).
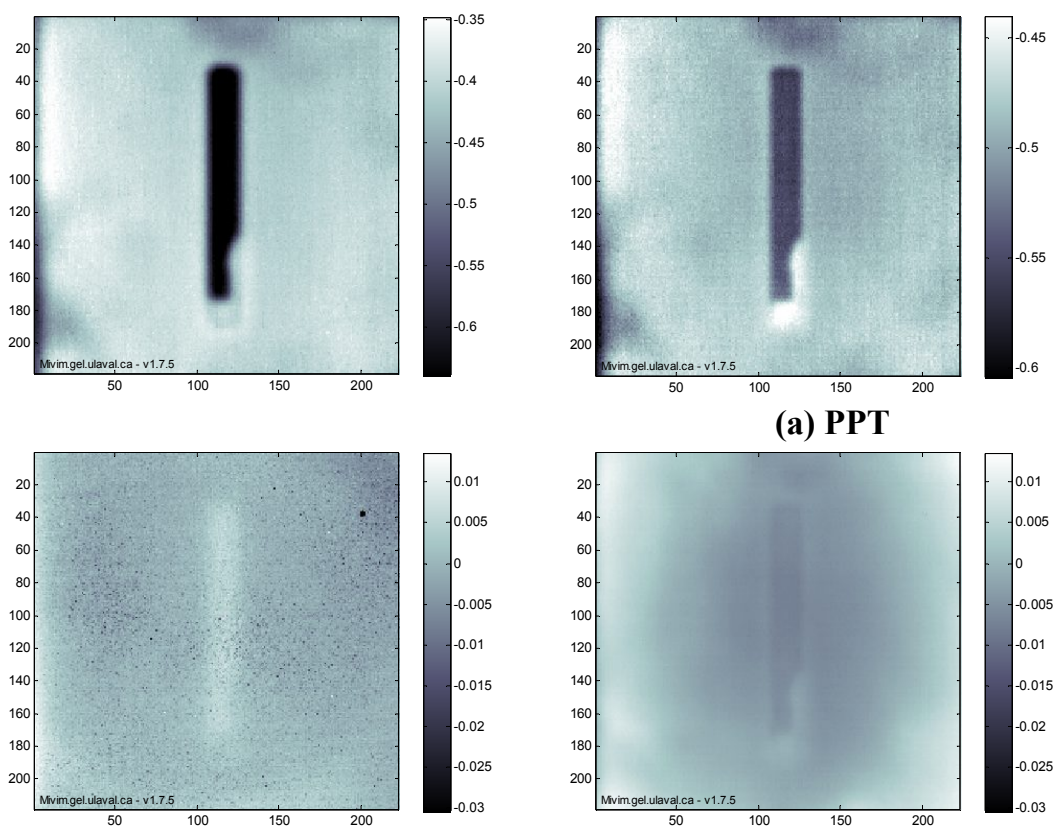

(b) PCT

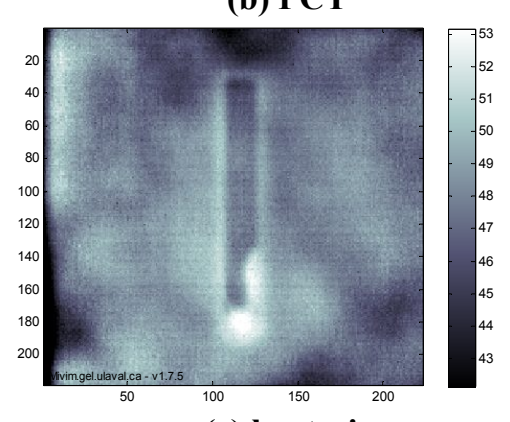

(c) kurtosis
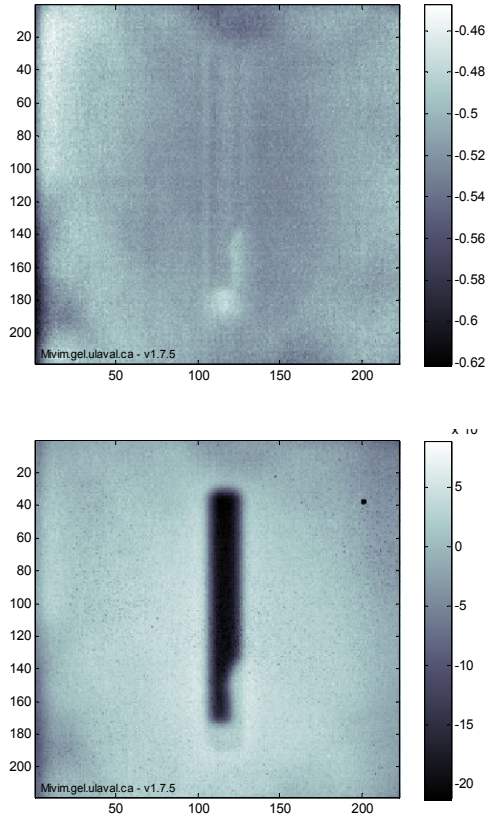

Figure 7. Images results of the processing of a Plexiglas ${ }^{\mathrm{TM}}$ specimen with (a) PPT (phasegram at $\mathrm{f}=.032 \mathrm{~Hz}$, (b) first three components obtained by principal component thermography (PCT), and (c) kurtosis analysis.

From the results in Figure 7 it is observed that it is possible to clearly identify the air and solder in the slab with the three techniques. However, PPT and PCT require the examination of the whole sequence, or at least a part of it, to determine the most suitable image for defect detection. On the contrary, the kurtogram in Figure $7 \mathrm{c}$ contains all the information about the defects. On the other hand, artifacts such as non-uniform heating are present in the kurtogram whilst they are 
considerably minimized in PPT, and they are present only in the first two PCT components but much less in the third. Still, the kurtosis method is very attractive for fully automated defect detection processing.

Future work will include the simulation of different types of defects and host materials and the validation with experimental data using academic specimens.

\section{CONCLUSIONS}

The proposed method based on the kurtosis statistic parameter has demonstrated to be a promising tool for detecting subsurface defect from pulsed thermography data. The kurtosis parameter calculation can be seen as a process to compress the thermographic sequence into a unique image, which exhibits enhanced contrast detection comparable to other well-known processing techniques in the field of pulsed thermography. An interesting characteristic of the kurtosis techniques is that it confers the possibility to process pulsed thermographic data in a completely automated matter. From simulated sequences, the performance of the techniques respect to depth and material diffusivity of defect has been checked. Experimental works using a modified Plexiglas ${ }^{\mathrm{TM}}$ specimen were performed More work has to be done in order to validate results using representative diffusivity and defect depth values.

Summarizing, the proposed technique provides an automatic method that can be used to produce compressed data (in a unique image) from large 3D matrices typical of active thermography experiments and enhanced contrast defection of defect material which diffusivity is higher than that of hosted material.

\section{ACKNOWLEDGEMENTS}

This work was supported by the Spanish Science and Technology Minister under project TEC2007-67987-C02-01 and José Castillejo program, the Chaire de recherche du Canada (MiViM) and the Ministère du développement économique, innovation et exportation du Québec.

\section{REFERENCES}

1. X. P. V. Maldague, "Theory and Practice of Infrared Technology for Nondestructive Testing" (WileyInterscience, April 2001).

2. R. E. Martin, A. L. Gyekenyesi, and S. M. Shepard, "Interpreting the results of pulsed thermography data", Materials Evaluation 61, 611 (2003).

3. C. Ibarra-Castanedo and X. Maldague, "Pulsed phase thermography reviewed",in Quantitative InfraRed Thermography (QIRT) Journal, 2004), Vol. 1, p. 47.

4. S. M. Shepard, J. R. Lhota, B. A. Rubadeux, D. Wang, and T. Ahmed, "Reconstruction and enhancement of active thermographic image sequences", Optical Engineering 42, 1337 (2003).

5. J. C. Ramirez-Granados, G. Paez, and M. Strojnik, "Dimensionless heat transfer model to compress and analyze pulsed thermography data for NDT of materials" in Thermosense XXX (SPIE, Bellingham WA, WA 982270010, United States, 2008), p. 69391.

6. S. Marinetti, L. Finesso, and E. Marsilio, "Matrix factorization methods: Application to thermal NDT/E", NDT\&E International 39, 611 (2006).

7. N. Rajic, "Principal component thermography for flaw contrast enhancement and flaw depth characterisation in composite structures", Composite Structures 58, 521 (2002).

8. F. J. Madruga, C. Ibarra-Castanedo, O. M. Conde, J. M. Lopez-Higuera, and X. Madalgue, "Automatic data processing based on the skewness statistic parameter for subsurface defect detection by active infrared thermography" in 9th International Conference in Quantitative InfraRed Thermography (QIRT), Lodz, 2008).

9. O. Holub and S. T. Ferreira, "Quantitative histogram analysis of images", Computer Physics Communications 175, 620 (2006).

10. "Thermocal 3D Software manual",in Institute of Introscopy, Tomsk Polytechnic, Russia, 1998).

11. C. Ibarra-Castanedo, "Quantitative subsurface defect evaluation by pulsed phase thermography:depth retrieval with the phase", 2005, Ph.D. Thesis, Laval University,[available online: http://www.theses.ulaval.ca/2005/23016/23016.pdf] 